\title{
Artículo \\ Deserción universitaria, una mirada desde la experiencia del Departamento de Atención Psicopedagógica $^{1}$
}

College dropout, a look from the Psychopedagogical Attention Department

Carmen Leticia Pérez Rodríguez

carmen.perez@udb.edu.sv

Resumen

En el presente artículo, la autora aborda el fenómeno de la deserción de estudiantes de la Universidad Don Bosco a través de las experiencias de los alumnos que buscan asistencia en el Departamento de Atención Psicopedagógica. El estudio se construye con la información recogida en las sesiones de los alumnos que han acudido a dicho departamento buscando algún tipo de ayuda durante 2014. Estos datos han servido más específicamente para dilucidar las causas de deserción y para diseñar un perfil emergente del estudiante desertor para dicha universidad.

Palabras clave: Deserción, estudiante, universidad, asistencia psicopedagógica
Abstract

In this paper, the author approaches dropout as a study case in Universdad Don Bosco students. This study is carried out through the experience of those students looking for assistance at the Psychopedagogical Attention Department. The study takes the data gathered from sessions of those students that by academic or personal reasons have looked for support at such department during 2014. These data have become the base to discover the dropout causes and to design a merging profile of the dropout student for this university.

Keywords: dropout, student, college, psychopedagogical attention

\footnotetext{
* La autora es Maestra en Diseño Currículo y colaboradora del Departamento de Atención Psicopedagógica de la Universidad Don Bosco, El Salvador.

1. Este artículo está basado en la ponencia "Deserción Universitaria, analizada desde la experiencia del Departamento de Atención Psicopedagógica de la UDB" que fue presentada en la Cuarta Conferencia Latinoamericana sobre el Abandono en la Educación Superior IV CLABES, celebrada los días 22, 23 y 24 de octubre de 2014 en la Universidad de Antioquia en Medellín, Colombia. 


\section{Introducción}

El presente artículo analiza el problema de la deserción universitaria a partir de la experiencia de los jóvenes que asisten al Departamento de Atención Psicopedagógica (DAP) de la Universidad Don Bosco (UDB) en San Salvador (El Salvador).

El Departamento de Atención Psicopedagógica (DAP) es la unidad académica de la Universidad que brinda apoyo, asistencia, orientación y seguimiento a las necesidades, intereses y problemáticas (bajo rendimiento, hábitos de estudio, problemas de aprendizaje, deserción) de los jóvenes universitarios que así lo demanden. Se encuentra dentro del campus principal en Soyapango (San Salvador) y es atendida por profesionales de psicología. El objetivo de DAP es brindar precisamente servicios de asistencia e intervención psicológica y psicopedagógica tanto a estudiantes como a otros miembros de la comunidad educativa.

La experiencia que a continuación se describe ha sido reconstruida con base a las sesiones de entrevista, terapia, evaluaciones diagnósticas y perfiles psicopedagógicos y de personalidad elaborados con el fin de analizar el problema de la deserción universitaria desde la experiencia de los jóvenes que asisten al DAP. Con ello además se ha buscado dilucidar las causas de la deserción universitaria, así como diseñar una versión emergente del perfil del estudiante que deserta y se retira de la Universidad. También se plantean algunas estrategias de intervención que la UDB ha desarrollado como propuestas para la disminución del índice de deserción.

\section{La deserción universitaria}

Para comprender el problema de la deserción comenzaré por su definición. Tinto y Giovagnoli (en Vásquez y otros, 2013) entienden la deserción como una situación a la que se enfrenta un estudiante cuando aspira y no logra concluir su proyecto educativo, considerándose como desertor a aquel individuo que siendo estudiante de una institución de educación superior no presenta actividad académica durante tres semestres académicos consecutivos.

Dentro de este concepto se aclara que es el alumno el que decide abandonar sus estudios; en este caso durante un año, por motivos diferentes a los académicos y de manera voluntaria. Además se pueden diferenciar dos tipos de abandonos en estudiantes universitarios: uno con respecto al tiempo y el otro referido al espacio. Con respecto al tiempo, puede hablarse entonces de (a) deserción precoz, individuo que habiendo sido aceptado por la universidad no se matricula; (b) temprana, individuo que abandona los estudios en los cuatro primeros semestres; y (c) tardía, individuo que abandona los estudios en los últimos seis semestres. En cuanto al espacio se puede hablar de (a) deserción institucional, cuando el estudiante abandona la universidad; (b) interna o del programa académico, cuando el alumno decide cambiar su programa académico por otro que ofrece la misma institución universitaria; y (c) 
la deserción del sistema educativo (Vásquez y otros, 2013). En la UDB se maneja la deserción en cuanto al espacio.

\section{Causas de la deserción en la UDB según los estudiantes}

La cobertura del DAP en el año 2013, según la Memoria de labores del DAP No 11 (2013), de Enero a Noviembre de 2013, se reporta que en cuanto a agenda programada, se brindaron 1435 consultas que beneficiaron a 1013 estudiantes. En en otros proyectos como el Curso de Inducción a la Vida Universitaria (CIVU) se evaluaron a 667 estudiantes de nuevo ingreso.

La mayoría de jóvenes beneficiarios del DAP llegan por iniciativa propia a buscar el apoyo del departamento; otros son referidos por docentes, tutores, coaches o directores de escuela. También existe un último grupo al que se le brinda servicios, que son estudiantes propios del Programa Persevera ${ }^{2}$, en este programa se focaliza la atención en el estudiante, sobre todo en aquel o aquella que se encuentra en riesgo de repitencia, deserción y/o reprobación académica, aunque se también se le brinda dicho servicio al estudiante que lo solicita.

En las sesiones que se tienen con los jóvenes que asisten al DAP, se identifica el problema de la deserción universitaria como un problema multicausal. La primera causa que se identifica es la situación de pobreza de la familiaque se vive en el país.

Según el Departamento de Gestión Social Estudiantil de la UDB (2014), ingresaron 7500 estudiantes a la Universidad durante el ciclo I-2014 (comprendido entre enero y mayo del año). Un $48.2 \%$ de de esos estudiantes provenían de las zona de influencia de la universidad (municipios de Soyapango, Apopa, Ciudad Delgado e llopango de San Salvador, constituidos mayormente por población de clase media baja); mientras que un 31.4\% provenía de municipios cercanos pero que no son de la zona de influencia; y el $20.4 \%$ restante proviene de otros municipio del país. Cabe mencionar también que de los estudiantes de nuevo ingreso en el ciclo I-2014, el 62.25\% provenía de instituciones educativas privadas, mientras que el $32.38 \%$ restante provenía de instituciones públicas. El $0.37 \%$ restante corresponde a estudiantes extranjeros.

Estos datos son importantes para comprender no sólo la caracterización de los estudiantes que ingresan a la Universidad, sino para comprender cómo la pobreza afecta la deserción. Otro dato igualmente importante es el ingreso per cápita de los estudiantes nuevos, así por ejemplo, durante este primer ciclo del año, el $89.9 \%$ percibe un ingreso per cápita ${ }^{3}$ en menor a un salario mínimo

2. El Programa Persevera está encaminado a mejorar los índices de eficiencia interna. Este programa tiene el coaching como componente del acompañamiento salesiano que se les brinda a los estudiantes. Es una una propuesta para la reducción de la repitencia y deserción.

3. Ingreso per cápita se calcula a través de la suma de ingreso familiares menos el egreso, dividido entre el número de miembros que conforman el grupo familiar. 
(\$224.10 USD); un 6.8\% corresponde a un ingreso per cápita entre \$224.10 USD y $\$ 320$ USD; mientras que el $3.4 \%$ restante percibe un ingreso mayor a los $\$ 320.01$ USD.

Como puede observarse en estos datos, la mayoría de los jóvenes provienen de familias donde el ingreso familiar es menor al salario mínimo del país, ello implica que no todos tienen la posibilidad de costearse un estudio universitario. Por ello a veces se retiran un tiempo, mientras trabajan y ahorran para obtener ingresos suficientes para reingresar y continuar sus estudios. Además hay casos en los que si comienzan a trabajar, disminuyen la carga académica que cursan. En lugar de cursar 5 o 6 asignaturas de su pensum por ciclo, solo inscriben 20 3. Eso implica que su plan de estudios de 5 años se alarga un poco más. En ocasiones esto los lleva a tomar decisiones como un cambio de carrera, por ejemplo, a estudios de nivel técnico universitario o algún profesorado, que son carreras de menor duración.

Otro de los factores que incide en este problema son las deficiencias académicas que los estudiantes tienen. Esto es evidente en las primeras asignaturas que cursan de las carreras. Un ejemplo de ello son los estudiantes del área de la Facultad de Ingeniería, quienes mencionan -y de hecho se puede constatar en el área de Registro Académico en el expediente de cada estudiante- que las mayores deficiencias están relacionadas a las áreas de Redacción y ortografía, Algebra lineal y Matemáticas.

De las consultas atendidas en el DAP, al menos 146 estudiantes se han acercado por problemas con sus hábitos de estudio o para que se les evalúe esa área. Hábitos de estudio es identificado como otro de los factores de deserción.

Al ingresar a la Universidad, en el Curso de Inducción a la Vida Universitaria (CIVU), el DAP evalúa a los jóvenes para elaborarles un perfil psicopedagógico de ingreso. Se evalúa a los futuros estudiantes en las áreas de aptitudes, intereses ocupacionales, hábitos de estudio y para completar dicho perfil se les pide que llenen un cuestionario sobre planes que ellos tienen para su futuro. En algunas de las facultades, como la de Aeronáutica, por el tipo de carreras que ofertan, también se evalúan la capacidad intelectual y aspectos de la personalidad.

Este perfil psicopedagógico es un insumo tanto para directores de escuela como para los docentes tutores quienes brindan un acompañamiento a los estudiantes a lo largo de su formación académica. El perfil les permite conocer las fortalezas del estudiante, así como aquellas áreas que deben desarrollar o potencializar más.

Cuando el estudiante siente que a pesar del tiempo que invierte en estudiar no está obteniendo los resultados esperados, se acerca para que se le evalúe en el área de hábitos de estudio, posterior a la evaluación se le entrega el respectivo informe, en el cual no sólo se identifican los malos hábitos puestos en práctica, sino también se elabora una propuesta de intervención educativa 
para mejorarlos y establecer nuevos hábitos y se les da el seguimiento necesario para obtener mejores resultados.

Una minoría de estudiantes se queja del docente y de las metodologías que emplean, sin asumir responsabilidad por los resultados académicos obtenidos. Ellos y ellas expresan que las evaluaciones son extensas, que muy poco tiempo se les otorga para realizar la evaluación y que las evaluaciones no están contextualizadas al contexto laboral con el que se enfrentarán al graduarse. Pero cuando se entrevista a otros estudiantes de las mismas carreras, afirman que se debe a los ritmos de trabajo y que tan organizados son para desarrollar la evaluación en el tiempo indicado. Por otra parte, cuando se aborda la misma problemática con estudiantes de niveles superiores, afirman que las evaluaciones si los preparan para el contexto laboral. Parece contradictorio, pero es cuestión de madurez profesional, al principio, las cosas no tienen sentido, luego, con el transcurso de su formación académica, se va comprendiendo el por qué el plan de estudios de la carrera ha sido diseñado.

Otra de las causas de deserción identificada por los jóvenes del DAP es la motivación intrínseca y extrínseca. Manifiestan que en ocasiones, dependiendo de la asignatura, las metodologías empleadas por el docente, resultan ser poco motivantes. Consideran que los contenidos abordados en el aula son monótonos y eso los desmotiva, sobre todo cuando ellos son bastante activos. A parte de eso se menciona la motivación intrínseca como otro de los factores porque en algunos casos los estudiantes optan por una carrera determinada por la presión económica o emocional en el hogar. El discurso, "si no estudias esta carrera, entonces no te pago la universidad" es muy frecuente en estos casos. Además puede verse la inmadurez emocional de los jóvenes para seleccionar una carrera universitaria, muchos de ellos se dejan llevar por la el nombre de la carrera o porque sus compañeros de Educación Media optaran por esa carrera, pero lo hacen sin investigar en qué consiste la carrera o sus campos de aplicación laboral. Otros llegan al DAP con sus padres para recibir una orientación vocacional sobre la carrera por la que pueden optar. Pero también hay un grupo minoritario que selecciona la carrera de acuerdo con la profesión de sus padres o en beneficio de los negocios familiares.

El último de los aspectos que pueden encaminar a los jóvenes a la deserción universitaria es el modelo de aprendizaje de los jóvenes actuales. Ellos están acostumbrados a la inmediatez tecnológica, todo lo quieren de forma inmediata y esperan que el docente les dé todas las herramientas para desempeñarse como profesionales, dejando de lado la construcción de su propio aprendizaje. A los jóvenes de hoy, podríamos catalogarlos según el Cuestionario de HoneyAlonso (Alonso, Gallego y García, 2006, 2009) de cómo estudiantes con Estilo de aprendizaje más pragmáticos, que se caracterizan por ser experimentadores, prácticos, directos, buscan no sólo la inmediatez, sino que lo que van a aprender realmente les resulte útil, buscan aprender de forma rápida y poner en práctica lo aprendido. En este sentido, buscan aprender cosas que les resulten significativas para la vida diaria. 


\section{Perfil psicopedagógico del estudiante que deserta en la UDB}

En el 2013 se brindaron 635 consultas en promedio por psicólogo, beneficiando a 312 estudiantes. De acuerdo con los perfiles psicopedagógicos y de personalidad de estos jóvenes atendidos, se ha elaborado el siguiente perfil (versión preliminar) de aquellos estudiantes que desertan de las carreras en la UDB.

Generalmente estos jóvenes presentan baja autoestima no sólo porque han crecido en un ambiente lleno de limitantes; sino también por la falta de educación de los padres. Esto no sólo se refiere a que los padres no tengan un nivel académico de estudio, sino más bien porque desconocen cómo el afecto, el tiempo de calidad y la estimulación temprana pueden favorecer el desarrollo de sus hijos y de como el maltrato, la violencia y los castigos pueden afectar el desarrollo de la autoestima. Usualmente, son jóvenes inseguros que no creen en sí mismos, aún cuando en los resultados de sus evaluaciones se evidencia la capacidad intelectual que tienen tanto para el estudio como para la carrera seleccionada. La inseguridad de estos jóvenes sumada a la carencia afectiva, los hace vulnerables a la opinión ajena de sus compañeros y docentes de aula y se vuelven fácilmente manipulables.

Además, en su mayoría son jóvenes con bajo perfil académico, ya sea por malos hábitos de estudio o por deficiencias académicas en la formación de Educación Media. Estos jóvenes ingresaron a las carreras y al primer obstáculo se frustran y dejan de ir a clases, hasta perder las asignaturas. Luego de perder varias asignaturas, una vez y otra vez, caen en la desesperación y optan por cambiar de carrera o irse de la universidad. Otra minoría tiene la capacidad para estar en las carreras y aprobar las asignaturas, pero pierden el tiempo y no se organizan con las actividades. Esta es otra de las características que presentan estos jóvenes, por ello sus trabajos reflejan poco de su capacidad intelectual, por su poca organización faltan a clases porque se quedan terminando tareas de otras asignaturas y eso les afecta al final del curso. Además se desaniman ante tareas difíciles y estudian según su humor.

Dentro del bajo perfil académico ha de mencionarse también el perfil aptitudinal de los estudiantes. En ocasiones no coincide el perfil del estudiante con la carrera seleccionada, por lo que se sugiere que luego de los resultados del primer ciclo si no son favorables o los que el estudiante espera, se considere un cambio de carrera. Pero la perseverancia o necedad del estudiante pueden llevarle a insistir en alguna asignatura para la que no tiene las aptitudes y le cuesta admitirlo hasta que ya no es posible continuar en dicha carrera.

La inmadurez emocional para afrontar las frustraciones en la carrera seleccionada es otra de las características de estos jóvenes, por lo general son ansiosos y muestran nerviosismo en las evaluaciones, trabajando apresuradamente y cometen errores por descuidos a la hora de responder en los exámenes.

Deserción universitaria, una mirada desde la experiencia del Departamento de Atención

42. Psicopedagógica
Otros jóvenes obtienen poco provecho en relación al tiempo de estudio. Probablemente se deba a los distractores con los que estudian (como música, 
redes sociales y televisión) o que memorizan sin comprender lo que estudian, también es evidente en que dudan en pedir explicaciones en el aula. Tienen una urgente necesidad por salir de la Universidad y comenzar a trabajar para lograr independencia económica y emocional de sus padres.

Cabe mencionar que otro aspecto importante es su falta de motivación porque aunque la mayoría está en carreras seleccionadas por su persona, no le encuentran el gusto a la carrera. Esperan que el docente les esté motivando en cada clase para mantener su atención e interés.

\section{Propuestas para disminuir la deserción}

Aunque la UDB desarrolla varios programas para disminuir los índices de deserción, repitencia y reprobación (Programa de Tutorías, de Instructorías, Persevera, Becas universitarias, Intervención psicopedagógica del DAP, entre otros). Para fines de este artículo, prestamos especial atención a la experiencia del DAP.

Intervención psicopedagógica en el DAP El DAP trabaja la intervención psicopedagógica que brinda bajo dos modalidades, una individual y otra colectiva. En cuanto a la atención individual, se trabajan tratamientos, asesorías psicológicas o evaluaciones para abordar las múltiples causas que afectan el rendimiento académico de los estudiantes. También dentro del DAP se considera el trabajar con estudiantes tutores como se hace a través del programa de becas de la universidad. Estos estudiantes tutores son jóvenes que se acercan al departamento con un buen rendimiento académico, pero con dificultades emocionales, al sobrellevarlas pueden ayudar a otros en las asignaturas que mejores calificaciones obtienen, convirtiéndose de esta forma en guía que acompaña a aquellos a los que se les dificulta una determinada materia.

Entre las causas por las que los jóvenes asisten podemos mencionar que la carrera seleccionada no concuerda con su perfil vocacional, dificultades familiares, dificultades de integración y/o adaptación, trastornos psicológicos no identificados (esto nos lleva en ocasiones a referirlos fuera de la UDB al área de psiquiatría, pero se les apoya con orientación o psicoterapia). Otros casos son referidos por sus tutores cuando han agotado sus recursos, pero en su mayoría asisten por iniciativa personal.

También se prestan servicios de evaluación y elaboración de perfiles de personalidad y carácter cuando ellos o ellas desean conocerse más a fondo y comprender por qué se comportan de una determinada manera.

En cuanto a la modalidad grupal, por una parte, se desarrollan talleres para estudiantes en temáticas de la psicología educativa previo diagnóstico con la unidad correspondiente. Estas temáticas pueden ser sobre motivación, actitudes y aptitudes para el estudio a la vida universitaria, entre otras. Por otra 
parte, también se realizan evaluaciones de aspirantes para distintos programas educativos como becas, Science Girl, Profesorados entre otros.

\section{Conclusión}

El problema de la deserción universitaria es un problema multicausal, pero la crisis económica no es el factor más fuerte que impulsa a los estudiantes a abandonar sus estudios, puesto que hay otras opciones como las becas universitarias.

De acuerdo con los datos de los estudiantes atendidos, consideramos que la principal causa de deserción universitaria es la falta de claridad del estudiante sobre lo que desea estudiar. También la presión de los padres por que estudien una carrera que les genere ingresos, que les permita hacerse cargo de los negocios familiares, que cumpla con las expectativas que los ellos tienen para con sus hijos son razones de peso. Pero la falta de madurez emocional para proyectarse hacia el futuro y verse dentro de su proyecto de vida, si son realmente importantes.

Hoy en día los jóvenes no saben lo que quieren hacer con su vida. Muchos solo viven el momento, y no piensan en el futuro y los que lo hacen, lo hacen de una forma fantasiosa e ingresan a carreras porque son nuevas y no son ofertadas en otras universidades del país porque el nombre de la carrera les parece atractivo y luego viene el fracaso cuando se dan cuenta que la carrera no es lo que habían pensado que sería.

Por ello es necesario que los jóvenes tengan una orientación vocacional oportuna, antes de ingresar a la universidad. En algunos de los colegios privados de educación media se tiene la oportunidad de contar con un departamento de psicología que les brinda este servicio. En otros casos, cuando las universidades visitan los centros educativos para hacer las ofertas educativas se les brinda este servicio, pero no se les profundiza sobre cada una de las carreras por lo que es indispensable que los jóvenes indaguen a profundidad sobre las aptitudes que requiere la carrera que han seleccionado el campo de trabajo, la malla curricular de la carrera, y otros aspectos.

Además es necesario trabajar en nivelar las deficiencias académicas que los jóvenes traen de la Educación Media. Esto puede hacerse a través del CIVU o un currículo paralelo que se implemente durante el primer semestre de estudio porque de no hacerlo, el primer ciclo será un fracaso. Probablemente no se obtengan los resultados esperados y no porque el estudiante no tenga la capacidad, sino porque sencillamente esas temáticas no fueron abordadas en su centro de estudios y esto los frustrará al ver que otros si responden y ellos no lo hacen. Los jóvenes tienden a comparar sus resultado con otros y esto puede llevarles a desertar de la carrera que habían seleccionado.

universitaria, una mirada desde la experiencia del Departamento de Atención 44. Psicopedagógica 
Otro aspecto importante es el trabajo conjunto con tutores. Se les brinda un acompañamiento continúo. El tutor y la escuela a la que pertenece el estudiante acompañan en la parte académica y el DAP se encarga de trabajar con ellos la parte emocional ya que es necesario que el joven tenga un equilibrio emocional para prestar la debida atención a sus estudios. Si ellos no están emocionalmente estables por problemas familiares, de pareja, de personalidad o autoestima, difícilmente podrán reflejar en las evaluaciones todo su potencial.

\section{Referencias}

Alonso, C.; Gallego, D. y García, J. (2006, 2009). Cuestionario Honey-Alonso de Estilos de Aprendizaje. [en línea]. info@estilosdeaprendizaje.com [fecha de consulta: 28 de Julio de 2014]. Disponible en: http://www.estilosdeaprendizaje.es/menuprinc2.htm

Departamento de Atención Psicopedagógica (2013). Memoria Mensual de Labores del DAP, No 11. Universidad Don Bosco. San Salvador.

Departamento de Gestión Estudiantil (2014). Informe de Caracterización Socioeconómica del Ciclo I-2014. Universidad Don Bosco: San Salvador.

Vásquez, J. y otros (2013). Informe final de investigación: Determinantes de la deserción estudiantil en la Universidad de Antioquia. Medellín. Colombia: Dirección de Bienestar Universitario. Vicerrectoría de Docencia. Centro de Investigaciones

Económicas.

45. Diálogos 